\title{
Carboxytherapy for Conservative Treatment of Peyronie's Disease
}

Muzi Fabrizio, D’Andria Daniele, Delicato Giampaolo, Baffigo Giulio, Tartaglia Edoardo, Corvese Francesco, Signore Stefano,Tati Eleonora, Perla Alessandro, Montagna Giuseppe and Tati Gaetano Department of Urology and Oncologic Urology, S. Eugenio Hospital, Rome, 00144, Rome, Italy

\begin{abstract}
PD (Peyronie's disease) is an acquired disorder of tunica albuginea characterized by the formation of plaques of fibrous tissue often associated to symptoms like ED (erectile dysfunction) and coital pain. The inflammatory process is unknown, even if it is known that Activated inflammatory cells produce many ROS (radical oxygen species), leading to fibroblast proliferation and collagen synthesis. Endothelian dysfunction is the responsible of infiammatory chain reaction in which an inflammatory protein, NF-kB seems involved in ROS synthesis. Conservative treatments (laser, ultrasound, iono/iontophoresis) seem to have poor therapeutic effects in PD. Clinical studies have indicated that altered $\mathrm{CO}_{2}$ (carbon dioxide) levels can impact upon disease progression. $\mathrm{CO}_{2}$ levels can be sensed by cells resulting in the initiation of pathophysiologic responses with a sensibile reduction of oxydative phenomena (Bohr/Haldane effects). We have tried carboxytherapy, by using sovrapubic subcutaneous injection of sterile $\mathrm{CO} 2$ gas in 20 patients aged from 40 to 65, affected by PD. After the cycle of treatment of 10 weekly applications. We have observed in all patients a subjective reduction of penile deviation, an improvement of quality of erections and a sensible reduction of plaque's dimensions, documented by ultrasound controls and IIEF questionary before and after the end of cycle. We should consider co2 as a powerful antioxidant against endothelian dysfunction and oxydative stress. NF-kB is a target of CO2 antioxidant power. Preliminary qualitative results could encourage an extended use of carboxytherapy in PD treatment.
\end{abstract}

Key words: Carboxytherapy, Peyronie's disease, induratio penis plastica, conservative treatment.

\section{Introduction}

PD (Peyronie's disease) is an acquired disorder of tunica albuginea characterized by penis plaques of fibrous tissue often associated with ED (erectile dysfunction).

PD (Peyronie's disease) affects $1-3 \%$ of the male population. Greater than $75 \%$ of patients with PD are between 45 and $65 \mathrm{y}$ of age. In this aging population the modulus of elasticity of tunical and septal tissues is diminished $[1,2]$.

Peyronie's disease has been linked to systemic conditions such as hypertension and diabetes.

PD is associated with Dupuytren's disease (palmar aponeurosis), Ledderhose's disease (plantar aponeurosis), Psoriasis.

Corresponding author: Fabrizio Muzi, M.D., specialist in general surgery and andrology, research field: urology and oncologic urology. E-mail: fabrizio.muzi@yahoo.it.
An immunologic component to Peyronie's disease has been proposed. Activated inflammatory cells produce oxygen radicals (ROS) initiating a chain of biochemical events leading to fibroblast proliferation and collagen apposition. The principal protein involved is NF-kB [1].

Carboxytherapy refers to the administration of $\mathrm{CO} 2$ for therapeutic purposes. It has been shown that $\mathrm{CO} 2$ has cell interaction in regulating factors of tissue perfusion. Nowadays $\mathrm{CO} 2$ is used as anti-aging treatment, for cellulite of all grades, for wrinkles reduction and for stretch marks [3].

Clinical studies have indicated that altered tissue $\mathrm{CO} 2$ levels can impact upon inflammation progression [4].

The objective of the study is to demonstrate that $\mathrm{CO} 2$ subcutaneous subministration, carboxytherapy, could obtain a sensibile reduction of fibrosis and oxidative phenomena of ROS in PD, reducing sexual 
discomfort of patients. Carboxytherapy could be employed as new easy method and free risk conservative treatment for PD.

\section{Material and Methods}

We have recruited 20 patients, aged from 40 to 60 years old, affected by PD until 1 year. Ten patients tried normal conservative therapy with ionophoresis and laser-ultrasound therapy without any results.

We have used IIEF questionary and we have studied ultrasound plaque's morphology before and after treatments to assess patients. We have injected by specific medical device $500 \mathrm{cc}$ of sterile Medical $\mathrm{CO}_{2}$ for 10-15 weekly applications in sovrapubic subcutaneous tissue. $\mathrm{CO}_{2}$ subcutaneous flux provokes a sort of subcutaneous emphysema along crural region, penis and scrotum. The controller regulates flux intensity and total volume of injection and is managed according to the sensibility of patients.

The medical device is externally connected to a $\mathrm{CO}_{2}$ sterile tank and provide to administrate the flux, heating the $\mathrm{CO}_{2}$ volume injected till human body temperature. The site of injection is the sovrapubic region, far from the plaque. We have used a 30 Gauge needle and a mean of 3 injections per application. $\mathrm{CO}_{2}$ has a subcutaneous diffusion along crural region, penis and using scrotus as reservoir. $\mathrm{CO}_{2}$ quantity could have a variation according to patient size. The subcutaneous emphysema is painless and disappears after 2-3 hours. The same method of $\mathrm{CO}_{2}$ subcutaneous sovrapubic application is already currently used in esthetic medicine, because $\mathrm{CO}_{2}$ has also special lipolithic properties.

\section{Results}

We have observed in all patients a subjective reduction of penile deviation, a reduction of penetration discomfort and an improvement of quality of erections. In four patients we have obtained the reduction of dimensions of plaques.

$\mathrm{CO}_{2}$ could be considered a powerful antioxidant against endothelian dysfunction and oxydative stress with easy management and poor toxicity.

In conclusion, $\mathrm{CO}_{2}$, as natural antioxidant could reduce the activity of inflammatory agents as NF-kB protein, involved in the evolution of $\mathrm{PD}$.

\section{Discussion}

The tunica albuginea is a thick fibroelastic sheath composed predominantly of thick collagenous bundles and elastic fibers surrounding the trabecular smooth muscle of the corpora cavernosa. Any defect in tunica albuginea can deform penile fibroelastic framework, resulting in curvature or bend, shortness, progressive lack of elasticity, and also occasionally affecting hemodynamic function of the penis $[1,2]$.

The inflammatory process origin is unknown. it is postulated the occurrence of penile trauma.

Injury causes delamination of the tunica albuginea predominantly at the dorsal, midline septum resulting in small hematoma $[4,5]$.

Inflammation, induration, and accumulation of leukocytes increase ROS activities with a chain of biochemical events leading to fibroblast proliferation and resulting in collagen and fibrin biosynthesis-deposition between the layers of the tunica albuginea [6].

It seems to be involved some specific inflammatory proteins that provoke the rising of radicals of oxygen and Nitric oxide. The principal protein involved in the genesis of ROS is NF-kB.

The nuclear transcription factor NF-kB is a heterodimeric, sequence-specific transcription factor found in many cells. In unstimulated cells, NF-kB is found in the cytoplasm and is bound to its inhibitor $\mathrm{kB}$, which prevents it from entering nuclei. A number of stimuli have been shown to activate NF-kB, including cytokines, activators of protein kinase $\mathrm{C}$, viruses and oxidants [7].

The family of NF-kappaB (nuclear factor kappaB) transcription factors is a topic of intense interest in the biomedical community stemming from the role 
NF-kappaB plays in almost every aspect of cell regulation: stress responses, immune cell activation, apoptosis, proliferation, differentiation and oncogenic transformation.

$\mathrm{NF}-\mathrm{\kappa B}$ has long been considered a prototypical proinflammatory signaling pathway, largely based on the activation of NF- $\mathrm{KB}$ by proinflammatory cytokines such as IL-1 (interleukin 1) and TNF $\alpha$ (tumor necrosis factor $\alpha$ ), and the role of NF-kB in the expression of other $\mathrm{p}$ roinflammatory genes including cytokines, chemokines, and adhesion molecules, which has been extensively reviewed elsewhere.

In PD altered $\mathrm{CO}_{2}$ levels could impact upon disease progression. $\mathrm{CO}_{2}$ levels can be sensed by cells resulting in the initiation of physiologic and pathophysiologic responses. A role for $\mathrm{CO}_{2}$ in the regulation of gene transcription has recently been identificated with exposure of cells and model organisms to high $\mathrm{CO}_{2}$ leading to suppression of genes involved in the regulation of innate immunity and inflammation.

Recent evidence suggests that $\mathrm{CO}_{2}$ may also directly regulate gene expression through the NF-kB pathway. The suppression of NF- $\mathrm{KB}$ activity by hypercapnia was recently provided by the demonstration of $\mathrm{CO}_{2}$-induced nuclear localization of the IKK $\alpha$ subunit [7].

Molecular $\mathrm{O}_{2}$ (oxygen) is sensed by prolyl and aspariginyl hydroxylases, which confer oxygen-dependent instability upon transcription factor. Activation of this pathway in hypoxia leads to the expression of adaptive genes. The sensor for regulation of NF- $\mathrm{kB}$-dependent gene expression in response to changes in $\mathrm{CO}_{2}$ has yet to be defined. However, elevated $\mathrm{CO}_{2}$ leads to repression of the NF- $\kappa \mathrm{B}$ pathway and decreased levels of genes which promote innate immunity and inflammation.

Therapeutic hypercapnia seems to have a suppressive effect on NF-kB signaling [7].

Carboxytherapy leads to flood vassels dilatation in the area where the gas is injected. This reaction to carbon dioxide injection gives a better oxygenation of skin layers with increased lipolysis capabilities. Carboxytherapy has been used for over 70 years in Europe where it was discovered (France, Royat). The procedure was widely used to treat ischemic vascular diseases because of the vasodilation properties of $\mathrm{CO}_{2}$. The most common $\mathrm{CO}_{2}$ treatments are: cellulite reduction, liposuction complementary solution, arterial diseases associated with diabetes and skin ulcers [3].

Carboxytherapy stimulates microcirculation at the level of metarterioles, arterioles and precapillary sphincteres by increasing tissue flow velocity and consequently, by improving lymphatic drainage $[3,8]$.

\section{Conclusion}

In PD the use of carboxytherapy shows in all patients subjective improvement of erection and in 15 cases the reduction of plaque dimensions.

Carboxytherapy shows very easy management and free risk subministration.Side effects are minimal and resolve quickly, and include mild pain at the injection site and possible self limiting bruising of the tissues.

Preliminary qualitative and quantitative results could encourage an extended experimentation of carboxytherapy in PD conservative treatment.

\section{References}

[1] Gelbard, M. K., Dorey, F., and James, K. 1990. "The Natural History of Peyronie's Disease." J. Urol. 144: 1376-80.

[2] Hellstrom, W. J. G., and Bivalacqua, T. 2000. "Peyronie's Disease: Etiology, Medical and Surgical Therapy-Review." J. Andrology 21: 347-54.

[3] Parassoni, L., and Varlaro, V. 1997. Carboxytherapy: A Method in Evolution. Rome: Editrice Salus Internazionale.

[4] Devine, C. J., Somers, K. D., and Ladaga, L. E. 1991. "Peyronie's Disease: Pathophysiology." Prog. Clin. Biol. Clin. Biol. Res. 370: 355-8.

[5] Somers, K. D., Sismour, E. N., and Wright, G. L. 1989. "Isolation and Characterization of Collagen in Peyronie's Disease.” J. Urol 141: 629-35.

[6] Schwarzer, U., Klotz, T., Braun, M. et al. 2000. "Prevalence of Peyronie's Disease-Results of an 8,000 Men Survey.” J. Urol. 163 (suppl): A742. 
[7] Taylor, C., and Cummins, E. 2011. "Regulation of Gene Expression by Carbon Dioxide.” Physiol. 15: 589 (Pt 4): 797-803.
[8] Parassoni, L.,Albergati, F.,Varlaro, V., and Curri, S. B. 1997. Carboxytherapy: Technique and Effects. Roma: Editrice Salus Internazionale. 